Revista de Psicología y Educación / Journal of Psychology and Education, 2021, 16(2), 104-124 (www.rpye.es) Doi: https://doi.org/10.23923/rpye2021.02.205

ISSN: $1699-9517$

\title{
Motivaciones y beneficios de participar en una experiencia educativa de aprendizaje y servicio con personas mayores en estudiantes universitarios jóvenes
}

\author{
Raquel Lorente-Martínez* , Esther Sitges-Maciá' , Natalia Lorente-Martínez² y Paula Brotons-Rodes \\ 1 Universidad Miguel Hernández de Elche \\ 2 Universidad de Granada
}

\begin{abstract}
Resumen: El voluntariado de personas mayores ha aumentado con la crisis sociosanitaria provocada por la COVID-19. En este trabajo se analizan las motivaciones del estudiantado universitario joven para participar en una acción educativa de voluntariado con personas mayores basada en la metodología de aprendizaje y servicio y se describen los beneficios que encuentran con su participación. Se ha realizado un diseño mixto anidado, con un estudio cualitativo primario pre-post, para conocer las motivaciones iniciales y las satisfechas del estudiantado universitario joven $(n=33)$ que participó en el Programa Acompaña-Té de la Universidad Miguel Hernández de Elche. A este estudio, se le anidó un estudio cuantitativo secundario al programa, para conocer la satisfacción, el interés en el voluntariado y la intención de participar en programas similares. Los conocimientos y los valores fueron las principales motivaciones para participar en el programa. Las participantes lograron mejorar sus conocimientos, desarrollaron su crecimiento personal, mejoraron su red social y reforzaron sus valores. La satisfacción con la formación de aprendizaje y servicio fue muy alta y todas manifestaron interés por realizar voluntariado en el futuro. Este tipo de formación contribuye a la educación integral del estudiantado universitario.

Palabras clave: Motivaciones, Personas mayores, Voluntariado, Aprendizaje y servicio, Educación superior.
\end{abstract}

\section{Motivations and benefits of participating in a service-learning educational experience with older adults among young university students}

\begin{abstract}
Volunteering with older adults has increased with the social and health crisis caused by COVID-19. This paper analyses the motivations of young university students to participate in an educational action of volunteering with older people based on the service-learning methodology and that describes the benefits they encounter in their participation. A mixed nested design was carried out, with a primary qualitative pre-post study, to determine the initial and satisfied motivations of young university students ( $n=33$ ) who participated in the Acompaña-Té Programme at the Miguel Hernández University of Elche. This study was followed by a secondary quantitative study to determinevolunteers' satisfaction, interest in volunteering and intention to participate in similar programmes. Knowledge and values were the main motivations for participating in the programme. Participants could improve their knowledge, develop their personal growth, improve their social network and strengthen their values. Satisfaction with the service-learning training was very high, and all participants expressed interest in volunteering in the future. This type of training contributes to the integral education of university students. Key words: Motivations, Older people, Volunteering, Service-learning, Higher education.
\end{abstract}

En el proceso educativo es preciso que el estudiantado adquiera conocimientos teóricos y prácticos necesarios para promover, entre otros, la educación para el desarrollo

Recibido: 16/03/2021 - Aceptado: 11/05/2021 - Avance online: 17/05/2021

*Correspondencia: Raquel Lorente Martínez

Universidad Miguel Hernández de Elche.

Dirección: 03202, Elche. España.

E-mail: rlorente@umh.es

Lorente-Martínez, R., Sitges-Maciá, E., Lorente-Martínez, N. y Brotons-Rodes, P. (2021). Motivaciones y beneficios de participar en una experiencia educativa de aprendizaje y servicio con personas mayores en estudiantes universitarios jóvenes. Revista de Psicología y Educación, 16(2), 104-124, https://doi.org/10.23923/rpye2021.02.205 sostenible y la ciudadanía mundial, como se defiende en el cuarto objetivo aprobado por la Organización de las Naciones Unidas (2015) en la Agenda 2030 sobre el Desarrollo Sostenible. Dentro de los desafíos a los que se enfrenta la ciudadanía global, el envejecimiento de la población supone uno ineludible, y sobre todo para los países más envejecidos como 
España (Pérez-Díaz et al., 2020). El plan de acción mundial sobre envejecimiento y salud establecido por la Organización Mundial de la Salud (2016) señala la necesidad de adoptar medidas educativas para combatir el fenómeno del edadismo y extender una visión más heterogénea de la vejez, que promueva el envejecimiento saludable, la igualdad de oportunidades de participación de todas las personas y la solidaridad entre generaciones.

Como afirman Ruiz-Corbella y BautistaCerro (2016, p. 185) "la universidad no puede permanecer ajena a la realidad en la que vive, sino que debe ser motor de desarrollo y cohesión social". Asumiendo su responsabilidad social, las universidades deben aceptar su papel protagonista en la formación superior integral en un marco de relación bidireccional con la sociedad, a través de la creación de iniciativas de formación, investigación, liderazgo social y compromiso social para hacer efectiva su incidencia en la sociedad (Beltrán-Llavador, Ínigo-Bajos y Mata-Segreda, 2014).

En esta línea, se ha considerado que los programas de innovación educativa y los programas de formación en voluntariado universitario son vías posibles para alcanzar este objetivo (Blesio y Mendoza, 2016), empleando metodologías como el aprendizaje y servicio para lograr una formación integral y educación superior de calidad (Gezuraga y García, 2020; Salazar, 2016). Esta metodología de aprendizaje sienta sus bases en la pedagogía experiencial de Dewey y el aprendizaje experiencial de Kolb y se caracteriza fundamentalmente por tres elementos: 1) integrar el aprendizaje académico con el servicio comunitario; 2) seguir una estrategia pedagógica basada en la experiencia; y 3) realizar un servicio orientado a atender una necesidad real de la comunidad. Además, otro elemento crucial en esta estrategia educativa es la reflexión crítica que el alumnado realiza sobre su experiencia de aprendizaje (Blesio y Mendoza, 2016; Gezuraga y García, 2020; Salazar, 2016).

Investigaciones educativas recientes señalan que los beneficios de este enfoque metodológico en estudiantes universitarios son múltiples y abarcan distintos niveles, entre los que destacan: el logro académico, la participación social y el desarrollo y crecimiento personal (Saz y Ramo, 2015). Los estudiantes encuentran este tipo de experiencias educativas muy enriquecedoras y satisfactorias, ya que les brindan la oportunidad de integrar los conocimientos teóricos y prácticos. Además, son experiencias transformadoras a nivel personal y profesional, puesto que contribuyen a mejorar su percepción personal, su conciencia social y sus competencias y habilidades profesionales (Caire, 2019; Gershfeld-litvin, 2020; Martínez et al., 2020). Asimismo, el servicio a la comunidad favorece la disminución de los estereotipos negativos, así como el aumento de la conciencia social y la intención de participar en acciones de voluntariado en el futuro (Brotons, Lorente y Sitges, 2020; Gershfeld-litvin, 2020; Lorente, Brotons y Sitges, 2020; Martínez et al., 2020; Primavera, 1999). También se han observado estos beneficios en estudiantes universitarios que participan en programas de voluntariado desarrollando estándares éticos y cualidades altruistas como el desinterés, la sensibilidad y la conciencia cívica (Popova y Popova, 2018) y mejorando el rendimiento académico, la autoestima y la motivación intrínseca hacia el trabajo académico (Johnson et al., 1998).

El perfil de los voluntarios de las ONGs en España muestra que la universidad es una de las principales vías de captación de voluntarios (Fundación Telefónica, 2019), siendo las áreas más representadas las Ciencias Sociales y Jurídicas y las Ciencias de la Salud, con acciones sociales dirigidas a la población infantil o grupos en riesgo de exclusión social (Fundación Mutua Madrileña, 2020). En general, se ha observado que existe una escasa oferta formativa de programas de voluntariado orientados hacia la población mayor en las universidades españolas (Sitges, Lorente y Saorín, 2017). Sin embargo, con la pandemia generada por la COVID-19 ha aumentado el número de voluntarios universitarios que han participado en programas dirigidos a la atención de personas mayores, pasando del $32 \%$ en el curso $2017-2018$ al $50 \%$ en el curso 2019-2020 (Fundación Mutua Madrileña, 2020). 
Para el desarrollo de este tipo de programas de voluntariado universitario, no solo se deben considerar las necesidades sociales que se van a atender, sino también los factores individuales que influyen en la implicación de los estudiantes en este tipo de acciones, esto es, conocer cuáles son sus motivaciones para participar en el voluntariado con personas mayores.

Desde el enfoque funcional propuesto por Clary y Snyder (1999) se han investigado las motivaciones hacia el voluntariado extensamente, entendiéndose que la conducta altruista se explica a través de seis motivaciones fundamentales, una de ellas orientada hacia los demás (valores) y cinco autodirigidas (mejora, social, carrera, protección y conocimiento). Además, se ha comprobado que estas motivaciones influyen en los procesos de captación de voluntarios, en la satisfacción de los voluntarios con la acción que realizan y en la intención de mantener la conducta voluntaria a lo largo del tiempo (Clary et al., 1998; Clary y Snyder, 1999).

Siguiendo este modelo, se ha observado que las motivaciones que impulsan a los universitarios a participar en programas de voluntariado son, de mayor a menor importancia: los valores, conocimientos, carrera, mejora, social y protección (Chapman y Morley, 1999; Francis, 2011 1). No obstante, se han encontrado diferencias generacionales en esta población. Por ejemplo, Francis (2011) comprobó que para la Generación Y -siguiendo a Schroer (2008) los nacidos entre 1977 y 1994, popularmente conocidos como "millennials"- era más importante la función social que para la Generación X (personas nacidas entre 1966 y 1976 que participaron en los estudios del equipo de Clary y colaboradores). Sin embargo, esta tendencia no se observó en estudiantes de la Generación Z (nacidos entre 1995 y 2012), en los que la función social ocupaba el último lugar de importancia (Cho, Bonn y Han, 2018). Además, Cho et al. (2018), fundamentando su estudio en la Teoría de la Acción Planeada, observaron que en esta generación las motivaciones autodirigidas hacia el desarrollo profesional, centradas en la carrera y la adquisición de conocimiento, eran mucho más relevantes que los valores orientados hacia la sociedad (situados en tercer lugar de importancia).

Para la evaluación de las motivaciones hacia el voluntariado Clary y Snyder (1998) diseñaron el Inventario de Funciones del Voluntariado que ha sido ampliamente utilizado en la literatura científica (Chacón et al., 2017; Chapman y Morley, 1999; Cho et al., 2018; Francis, 2011; Stukas et al., 2016). Este instrumento ha sido criticado porque limita a seis las motivaciones y sobreestima el número de motivos que tienen los voluntarios, por lo que se ha sugerido combinar la evaluación cuantitativa con la cualitativa, a través de preguntas abiertas, para conocer las verdaderas motivaciones de los voluntarios (Chacón et al., 2010; Francis, 2011; Vecina y Marzana, 2019). Desde este nuevo enfoque, Vecina y Marzana (2019) han propuesto una nueva clasificación, añadiendo seis motivaciones más al modelo funcional, lo que suma un total de 12 motivaciones hacia el voluntariado, cuatro orientadas hacia los demás (valores, preocupación comunitaria, compromiso social y reciprocidad) y ocho autodirigidas (mejora, conocimiento, carrera, protección, social, diversión, crecimiento personal e interés en la tarea).

Otro aspecto relevante en el estudio de las variables que explican la permanencia en las organizaciones de voluntariado ha sido la satisfacción. Se ha propuesto un modelo de tres factores de la satisfacción (motivaciones, organización y tarea), que explica una parte de la intención de continuar en el voluntariado y, a su vez, predice la permanencia en las organizaciones de voluntariado (Jiménez, Fuertes y Abad, 2009). De este modo, la relación entre las motivaciones de los participantes y la satisfacción de estas, así como otros aspectos organizativos, se deberían considerar en la formación del voluntariado universitario.

Con el aumento de la oferta de programas de voluntariado universitario con personas mayores, resulta fundamental conocer las motivaciones que impulsan al estudiantado a 
participar en estos programas y los beneficios funcionales que obtienen al hacerlo, puesto que la evidencia científica ha demostrado que las motivaciones hacia el voluntariado y la satisfacción de las mismas a través de la experiencia con el voluntariado influyen en la satisfacción y la intención de participar en estas acciones en el futuro (Clary et al., 1998; Clary y Snyder, 1999; Jimenez, Fuertes y Abad, 2009). Además, el análisis de estas cuestiones permitirá diseñar programas de formación en voluntariado universitario que se ajusten a las necesidades del alumnado joven y ofrezcan experiencias de aprendizaje integral que motiven a estos jóvenes a implicarse en acciones de voluntariado posteriormente, creando así sociedades más solidarias y comprometidas. Hasta donde alcanza nuestro conocimiento, el voluntariado de adolescentes y jóvenes ha sido poco investigado (Francis, 2011 ; Johnson et al., 1998) y no se han realizado investigaciones en este sentido. Esta investigación pretende cubrir este vacío, al tratar de conocer las motivaciones que impulsan al estudiantado universitario joven (Generación Z) a formarse en programas de voluntariado de personas mayores y los beneficios que obtienen al participar en una experiencia de aprendizaje y servicio de acompañamiento a personas mayores que viven solas. Para ello se plantean los siguientes objetivos e hipótesis:

El primer objetivo es analizar cuáles son las motivaciones que impulsan al estudiantado universitario joven a participar en una formación de voluntariado con personas mayores (Programa Acompaña-Té). La primera hipótesis del estudio, siguiendo los hallazgos de Cho et al. (2018) con estudiantes de la Generación Z, es que el estudiantado universitario joven que se inscriba en esta formación de voluntariado lo hará por motivaciones autodirigidas orientadas al desarrollo profesional (por ej. carrera y conocimientos), más que por motivaciones orientadas hacia los demás (por ej. valores) y, además, la función social del voluntariado no será relevante para motivar su participación. Asimismo, siguiendo a Vecina y Marzana (2019), estos jóvenes referirán pocas motivaciones y estas serán más variadas que las propuestas por en el modelo tradicional de seis factores.

El segundo objetivo del estudio es describir las motivaciones del estudiantado universitario joven que se satisfacen al participar en el Programa Acompaña-Té. Teniendo en cuenta los ejes principales del programa (la educación sobre la soledad en la vejez, la sensibilización hacia los problemas que afectan a las personas mayores y el acompañamiento de personas mayores que viven solas), la segunda hipótesis del estudio es que el estudiantado joven que participe en esta acción satisfará las motivaciones relacionadas con la adquisición de conocimientos, de valores de solidaridad hacia las personas mayores y de mejora por la creación de nuevas relaciones sociales.

El tercer y último objetivo de este trabajo es analizar la satisfacción de las motivaciones iniciales del estudiantado universitario joven que ha participado en esta formación e integrar esta información con el nivel de satisfacción que tienen con el programa de formación, la intención de participar en programas similares con personas mayores y el interés de participar en acciones de voluntariado. Teniendo en cuenta la evidencia acumulada desde la aproximación funcional del voluntariado (Clary et al., 1998; Clary y Snyder, 1999), la tercera hipótesis es que los voluntarios que obtengan beneficios funcionalmente relevantes al participar en el programa estarán más satisfechos con el programa en general y tendrán intención de participar en acciones de voluntariado similares e interés en el voluntariado.

\section{MÉTODO}

\section{DISEÑO}

Se ha llevado a cabo un diseño mixto anidado (Escalante-Barrios et al., 2020), con un estudio cualitativo primario, con diseño pre-post, para conocer las motivaciones iniciales del estudiantado universitario joven para participar en un programa de formación en voluntariado de personas mayores y las motivaciones que satisficieron tras participar 
en el mismo. A este estudio, se le anidó un estudio cuantitativo secundario secuencial al finalizar el programa realizado en los mismos participantes, para conocer la satisfacción con su experiencia y la intención e interés por participar en el futuro en actividades de voluntariado.

\section{PARTICIPANTES}

De un total de 40 estudiantes de la Universidad Miguel Hernández de Elche $(\mathrm{UMH})$, que participaron en las dos ediciones del Programa Acompaña-Té desarrolladas durante los cursos académicos 2015-2016 y 2017-2018, se seleccionó incidentalmente a las personas jóvenes de este grupo (menores de 30 años) que habían completado el programa. Quedaron excluidas del estudio cinco participantes de 45 o más años que sí completaron el programa y dos participantes de 30 o menos años que solo realizaron la parte teórica. De las 33 personas voluntarias jóvenes que completaron la formación, 32 eran estudiantes de grado y una estudiante de máster. El 84,8\% fueron mujeres (por lo que nos referiremos en femenino al grupo de participantes a partir de ahora en el informe), con edades comprendidas entre 18 y 28 años $(M=20 ; D T=2,15)$, donde el $87,8 \%$ eran de la Generación Z, en este caso estudiantes universitarias nacidas entre los años 1995 y 2000. El 33,3\% de las participantes tenía experiencia previa de voluntariado, acumulando una experiencia de voluntariado en meses que oscilaba entre los tres y los 24 meses, con una media de duración del voluntariado de 3,3 meses $(D T=6,4)$. En la Tabla 1 se presenta una descripción del perfil de las participantes.

Tabla 1

Características de las participantes del estudio

\begin{tabular}{|c|c|c|c|}
\hline & & Frecuencia (f) & Porcentaje (\%) \\
\hline \multirow[t]{9}{*}{ Estudiantes de Grado } & Titulaciones & 32 & 97 \\
\hline & Ciencias Políticas & 1 & 3 \\
\hline & Comunicación Audiovisual & 2 & 6,1 \\
\hline & Derecho & 3 & 9,1 \\
\hline & Ingeniería en Biotecnología & 1 & 3 \\
\hline & Ingeniería en Telecomunicaciones & 1 & 3 \\
\hline & Periodismo & 6 & 18,2 \\
\hline & Psicología & 14 & 42,4 \\
\hline & Terapia Ocupacional & 5 & 15,2 \\
\hline Estudiantes de Máster & $\begin{array}{c}\text { Gestión de Recursos Humanos, Trabajo y } \\
\text { Organizaciones }\end{array}$ & 1 & 3 \\
\hline \multirow[t]{5}{*}{ Curso académico } & $1^{\circ}$ & 18 & 54,5 \\
\hline & $2^{\circ}$ & 8 & 24,2 \\
\hline & $3^{\circ}$ & 5 & 15,2 \\
\hline & $4^{\circ}$ & 1 & 3 \\
\hline & Máster 60 ECTS (curso único) & 1 & 3 \\
\hline
\end{tabular}


Tabla 1 (Continuación)

Características de las participantes del estudio

\begin{tabular}{|c|c|c|c|}
\hline \multicolumn{2}{|c|}{} & Frecuencia (f) & Porcentaje (\%) \\
\hline $\begin{array}{c}\text { Experiencia previa de } \\
\text { voluntariado }\end{array}$ & No & 22 & 66,7 \\
\cline { 2 - 4 } & Si & 11 & 33,3 \\
\hline \multirow{2}{*}{$\begin{array}{c}\text { Duración total del } \\
\text { voluntariado }\end{array}$} & 3 meses & 1 & 3,0 \\
\cline { 2 - 4 } & 4 meses & 3 & 6,1 \\
\cline { 2 - 4 } & 5 meses & 1 & 9,1 \\
\cline { 2 - 4 } & 7 meses & 3 & 3,0 \\
\cline { 2 - 4 } & 18 meses & 1 & 9,1 \\
\hline
\end{tabular}

\section{PROGRAMA DE INTERVENCION}

El Programa Acompaña-Té es un programa bienal de formación en voluntariado universitario con personas mayores basado en el enfoque pedagógico del aprendizaje experiencial y en el que se emplea una metodología de enseñanza de aprendizaje y servicio. El programa se ofrece desde el servicio de extensión universitaria a toda la comunidad universitaria. El curso se desarrolla durante 50 horas de formación organizadas en dos partes: una teórica, grupal, y una práctica, de acompañamiento domiciliario a una persona mayor que vive sola en la comunidad. La parte teórica consta de 25 horas, distribuidas en seis sesiones de cuatro horas en las que se exponen los siguientes contenidos: sesión 1, la soledad en la vejez; sesión 2, el edadismo y su influencia en las personas mayores y la soledad; sesión 3, factores individuales de la persona mayor y la persona voluntaria que influyen en el acompañamiento; sesión 4, factores sociales y de la intervención que influyen en el acompañamiento; sesión 5, el voluntariado con personas mayores; y sesión 6, la guía para el acompañamiento de personas mayores que viven solas. En estas sesiones se emplean metodologías participativas y se realizan dinámicas grupales de presentación y de análisis y profundización ( $p$ ej. el "árbol de los prejuicios" para trabajar sobre el edadismo y juegos de roles para representar el manejo de situaciones difíciles durante el acompañamiento). La formación teórica finaliza con una actividad de reflexión sobre los contenidos vistos durante las sesiones y la presentación de la guía de acompañamiento. La parte práctica está constituida por una intervención psicosocial de acompañamiento de nueve sesiones de dos horas y media de duración, distribuidas semanalmente, en la que se realizan actividades dentro y fuera del domicilio de la persona acompañada. Al concluir el programa las participantes deben realizar una actividad de reflexión final y valorar distintos aspectos del programa.

\section{INSTRUMENTOS DE MEDIDA}

Se utilizaron diferentes instrumentos de medida para recoger las variables cualitativas (motivaciones iniciales y satisfechas) y cuantitativas (satisfacción, intención e interés con el voluntariado) que se analizan en este estudio.

Los datos cualitativos se recogieron con diferentes instrumentos en tres momentos temporales del estudio (Tabla 2). Para la evaluación pre-intervención se diseñó un formulario de Google ${ }^{\circledR}$ que recogía datos sociodemográficos, disponibilidad geográfica 
y temporal, experiencia previa de voluntariado y motivaciones iniciales para participar en el programa. Para la evaluación postintervención se diseñaron dos instrumentos: 1) la memoria de actividades y 2) el cuaderno de evaluación final, de los que se extrajeron las motivaciones satisfechas con el Programa Acompaña-Té.

Los datos cuantitativos se recogieron en el cuaderno de evaluación final. Por un lado, se evaluó el grado de satisfacción global con el Programa Acompaña-TÉ; el grado de satisfacción con el acompañamiento realizado; y el grado de satisfacción con la coordinación del programa mediante preguntas ad-hoc con una escala tipo Likert de 10 puntos (donde "1" significa "nada satisfecho" y "10" significa "muy satisfecho"). Para estimar el nivel de satisfacción de las motivaciones de las participantes, se comparó la correspondencia entre las motivaciones iniciales y aquellas satisfechas al final del programa, cuando había al menos una correspondencia entre ambas se consideró satisfecha la motivación. Por otro lado, mediante una escala de respuesta dicotómica (si/no), se evaluó si tenían interés en realizar algún tipo de voluntariado en el futuro y, específicamente, si tenían intención de realizar voluntariado de acompañamiento con personas mayores.

\section{PROCEDIMIENTO}

La recogida de datos se realizó durante las dos ediciones celebradas del Programa en 2016 y 2018, en tres momentos temporales. En la primera fase (pre-inscripción) las personas interesadas cumplimentaron el formulario de Google ${ }^{\circledR}$. Se recibieron un total de 85 solicitudes entre las dos ediciones

Tabla 2

Recogida de datos cualitativos

\begin{tabular}{|c|c|c|}
\hline Fase & Instrumento & Preguntas \\
\hline Pre-inscripción & Formulario online & $\begin{array}{l}\text { ¿Por qué te gustaría participar en este programa de formación } \\
\text { en voluntariado con personas mayores? }\end{array}$ \\
\hline Tras finalizar formación teórica & Memoria de actividades & $\begin{array}{l}\text { ¿Qué has aprendido del curso de voluntariado específico para } \\
\text { el acompañamiento de personas ancianas que viven solas? }\end{array}$ \\
\hline \multirow{5}{*}{$\begin{array}{l}\text { Tras finalizar programa } \\
\text { (teoría + acompañamiento) }\end{array}$} & \multirow{5}{*}{ Cuaderno de evaluación final } & $\begin{array}{l}\text { ¿Qué has aprendido realizando el acompañamiento de la } \\
\text { persona asignada en el Programa Acompaña-Té? }\end{array}$ \\
\hline & & $\begin{array}{c}\text { ¿Cómo te sientes después de haber llevado a cabo el } \\
\text { acompañamiento de la persona asignada en el Programa } \\
\text { Acompaña-Té? }\end{array}$ \\
\hline & & $\begin{array}{c}\text { ¿Qué ha sido lo más positivo de realizar el Programa Acompaña- } \\
\text { Té? }\end{array}$ \\
\hline & & $\begin{array}{c}\text { ¿Qué ha sido lo más negativo de realizar el Programa } \\
\text { Acompaña-Té? }\end{array}$ \\
\hline & & ¿Te ha transformado de alguna manera esta experiencia? \\
\hline
\end{tabular}


y la coordinadora seleccionó telefónicamente 20 personas por edición. En la segunda fase, tras realizar la formación teórica y antes del acompañamiento, las participantes tuvieron que cumplimentar y entregar de manera electrónica la memoria de actividades a la coordinadora. En la tercera fase, una vez recibida la formación teórica y realizado el servicio de acompañamiento, las participantes cumplimentaron en una sesión individual, sin la presencia de ninguna investigadora, el cuadernillo de evaluación final de papel y lápiz en el que reflexionaron sobre su experiencia de aprendizaje y valoraron el programa.

La formación teórica fue impartida en una sala diáfana y multifuncional de la UMH en seis sesiones organizadas los viernes por la tarde y los sábados por la mañana durante los meses de marzo y abril de 2016 y 2018. La coordinadora del programa y una ayudante impartieron las sesiones y manejaron las dinámicas grupales y para los contenidos específicos de voluntariado se invitó a una organización del tercer sector en cada edición (Cruz Roja y Fundación DASYC respectivamente). Las sesiones prácticas se organizaron entre los meses de mayo, junio y julio y durante las mismas la coordinadora y su ayudante estaban disponibles por teléfono para aclarar cualquier cuestión o problema que pudiera surgir.
Este proyecto obtuvo la aprobación del comité de ética de la UMH (Ref. DPS.ESM.01 . 16) y contó con el consentimiento informado de las participantes y su autorización para el uso de los datos con fines académicos y de investigación, garantizando su anonimato y protección, como establece el procedimiento ético de la Declaración de Helsinki y la Ley Orgánica 15/1999 de Protección de Datos de Carácter Personal vigente en el momento del estudio.

\section{ANÁLISIS DE DATOS CUALITATIVOS}

Todos los datos cualitativos recogidos se seleccionaron y agruparon en 33 documentos primarios que formaron la unidad hermenéutica de análisis del estudio. Para extraer y analizar las motivaciones iniciales y las satisfechas se realizó un análisis temático semántico (explícito/ literal) y guiado teóricamente (deductivo) de las narrativas de las participantes, siguiendo las recomendaciones de Braun y Clarke (2006). Se buscaron en los discursos 12 temas/motivaciones hacia el voluntariado (Tabla 3), basados en los planteamientos de la aproximación funcional del voluntariado (Clary et al., 1998; Clary y Snyder, 1999) y en la revisión y propuesta realizada por el equipo de Fernando Chacón y María Luisa Vecina (Chacón et al., 2010, 2017; Vecina y Marzana, 2019).

Tabla 3

Motivaciones hacia el voluntariado (extraído de Vecina y Marzana, 2019, p. 577-578)

\begin{tabular}{|c|c|c|}
\hline Tipo de motivo & Motivación & Descripción \\
\hline \multirow{4}{*}{$\begin{array}{l}\text { Orientadas a los demás } \\
\text { (hetero-motivadas) }\end{array}$} & Valores & $\begin{array}{c}\text { Expresar valores relacionados con preocupaciones altruistas } \\
\text { y humanitarias en general. Se incluyen en esta categoría los } \\
\text { valores religiosos de ayuda al prójimo. }\end{array}$ \\
\hline & Preocupación comunitaria & $\begin{array}{l}\text { Interés en ayudar de manera particular a un grupo de } \\
\text { población o en una localidad específica. }\end{array}$ \\
\hline & Compromiso social & $\begin{array}{l}\text { Expresar valores ideales que subrayan la necesidad de un } \\
\text { cambio social para reducir la injusticia y mejorar el mundo. }\end{array}$ \\
\hline & Reciprocidad & $\begin{array}{l}\text { Expresiones referidas a la norma social de reciprocidad, en las } \\
\text { que se hace explícito el deseo de pagar o devolver las cosas } \\
\text { que se han recibido en el pasado. }\end{array}$ \\
\hline
\end{tabular}


Tabla 3 (Continuación)

Motivaciones hacia el voluntariado (extraído de Vecina y Marzana, 2019, p. 577-578)

\begin{tabular}{|c|c|c|}
\hline Tipo de motivo & Motivación & Descripción \\
\hline \multirow{8}{*}{$\begin{array}{l}\text { Orientadas hacia uno mismo } \\
\text { (auto-motivadas) }\end{array}$} & Mejora & $\begin{array}{c}\text { Expresiones centradas estrictamente en mejorar la autoestima } \\
\text { personal y/o hacer nuevos amigos. }\end{array}$ \\
\hline & Conocimiento & $\begin{array}{l}\text { Expresiones que aluden a que el voluntariado permite adquirir } \\
\text { nuevas experiencias de aprendizaje y la oportunidad de poner en } \\
\text { práctica conocimientos, habilidades y competencias que de otro } \\
\text { modo no practican. }\end{array}$ \\
\hline & Carrera & $\begin{array}{l}\text { Expresiones que manifiestan la intención de ganar experiencia } \\
\text { relacionada con la carrera a través del voluntariado }\end{array}$ \\
\hline & Protección & $\begin{array}{c}\text { Expresiones centradas en proteger el ego de características } \\
\text { negativas personales, como sentimientos de culpa o problemas } \\
\text { personales. }\end{array}$ \\
\hline & Social & $\begin{array}{l}\text { Expresiones que aluden a la relación con otras personas y } \\
\text { manifiestan que realizan estas actividades porque son vistas de } \\
\text { manera favorable por personas importantes para ellas. }\end{array}$ \\
\hline & Disfrute & Expresar la necesidad de pasarlo bien y disfrutar. \\
\hline & Crecimiento personal & $\begin{array}{c}\text { Expresiones que destacan la oportunidad del voluntariado para } \\
\text { mejorar de manera positiva personalmente y para crecer como } \\
\text { personas. }\end{array}$ \\
\hline & Interés en la tarea & $\begin{array}{l}\text { Expresiones centradas en el interés en la actividad de voluntariado } \\
\text { en cuestión, no en el voluntariado o sus objetivos. }\end{array}$ \\
\hline
\end{tabular}

El procedimiento seguido para el análisis de datos cualitativos fue el siguiente. Una investigadora, instruida en las motivaciones hacia el voluntariado, realizó la segmentación de los documentos primarios seleccionando las citas vinculadas con los temas (códigos) acordados previamente. Posteriormente, dos investigadoras del equipo se encargaron de identificar de manera independiente cada cita seleccionada con los códigos (Tabla 3). Las discrepancias encontradas en esta codificación se resolvieron mediante discusión entre las cuatro investigadoras. La concordancia interiveces se midió con el índice kappa de Cohen, siguiendo los criterios de Landis y Koch (1977) para su interpretación, mostrando una fiabilidad casi perfecta entre las investigadoras ( $k=, 90$ investigadora R.L. y $k=, 85$ investigadora N.L.).

Todos los temas detectados en el discurso de las participantes se analizaron posteriormente con la técnica de análisis de contenido, para describir de forma cuantitativa los resultados encontrados, y, finalmente, con la técnica del análisis temático se analizaron las citas vinculadas a cada código de las motivaciones satisfechas, para profundizar en el conocimiento de los beneficios que encontraron las participantes del estudio al realizar el programa y proponer, si procediera, subcódigos temáticos. Dichos análisis se realizaron mediante el software informático de análisis de datos cualitativos ATLAS.ti versión 7.5.7.

\section{ANÁLISIS DE DATOS CUANTITATIVOS}

Para el análisis de los datos cuantitativos se realizaron análisis descriptivos con medidas de tendencia central y dispersión (medias y desviaciones típicas) en las variables continuas y frecuencias absolutas y relativas en las 
variables categóricas. Los datos recogidos fueron registrados y codificados mediante el programa IBM SPSS Statistics versión 27.0.

\section{RESULTADOS}

\section{MOTIVACIONES INICIALES PARA PARTICIPAR EN EL PROGRAMA}

Un total de 58 citas fueron identificadas como motivaciones iniciales para participar en el programa, siendo entre uno y tres los motivos referidos para participar $(M=1,69$; $D T=0,72)$. La mayoría de participantes mencionó un único motivo $(45,5 \%)$, seguidas por las que mencionaron dos y tres motivos (39,4\% y $15,2 \%$ respectivamente).

En la Figura 1 se observan las motivaciones iniciales referidas con mayor frecuencia, que de mayor a menor fueron: conocimiento $(29,3 \%)$, valores $(19 \%)$, crecimiento personal (13,8\%), preocupación comunitaria $(12,1 \%)$ y carrera $(10,3 \%)$. Con menor frecuencia que las anteriores, también se mencionaron motivaciones como el disfrute (5,2\%), mejora, interés en la tarea y compromiso social $(3,4 \%$ respectivamente). Los motivos de protección, social y reciprocidad no fueron referidos por ninguna participante al inscribirse en el programa.

\section{MOTIVACIONES SATISFECHAS CON EL PROGRAMA}

Se identificaron 193 citas sobre motivaciones o beneficios obtenidos con la participación en el programa. Como se puede observar en la Tabla 5, el número de motivaciones satisfechas fue mayor que el de motivaciones iniciales con una media de $3,3(D T=0,95)$, con un mínimo de una y un máximo de cinco motivaciones satisfechas.

La principal motivación que lograron satisfacer las participantes fue la de conocimiento con un $52,8 \%$ de citas. Le siguieron, de mayor a menor, las motivaciones de crecimiento personal (16,6\%), mejora (15\%) y valores $(5,2 \%)$. Con menor frecuencia también lograron satisfacermotivaciones de compromiso social (2,6\%), preocupación comunitaria, reciprocidad $(2,1 \%$ respectivamente), carrera y disfrute (1,6\% respectivamente). En este caso tampoco la motivación protectora y social fueron mencionadas por las participantes.

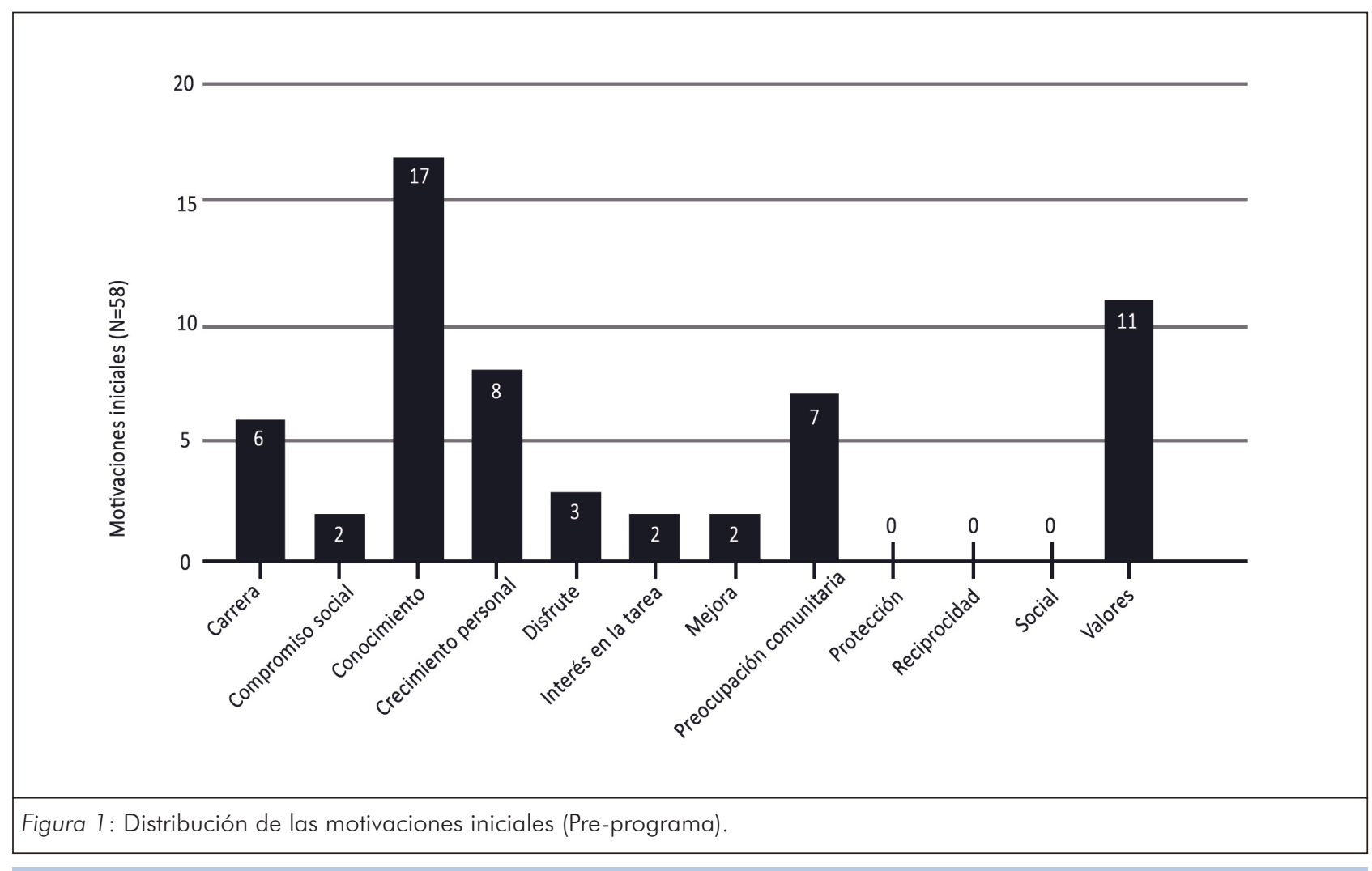




\section{ANÁLISIS TEMÁTICO DE LAS MOTIVACIONES SATISFECHAS POR LAS PARTICIPANTES}

En la Tabla 4 se presentan los temas (códigos y subcódigos) que han aparecido de forma sistemática en las narrativas de las participantes y, a continuación, se describen aquellos que fueron mencionados con mayor frecuencia, para comprender mejor cuáles fueron los beneficios reportados por las participantes.

CONOCIMIENTO. Esta fue la motivación más citada por las participantes, tanto al inicio, donde 16 participantes en 17 citas reconocieron que buscaban aprender y vivir nuevas experiencias, como al final del programa, donde todas las participantes excepto una mencionaron el conocimiento como una de sus motivaciones satisfechas. Tres subtemas principales se han encontrado:

SENSIBILIZACIÓN HACIA LA VEJEZ. En general reconocen haber aumentado su conocimiento sobre el envejecimiento y los desafíos que enfrentan las personas mayores $y$, en particular, destacaron el conocimiento sobre el fenómeno del edadismo y su detección en el entorno inmediato.

'Aquí, he podido ver que las personas mayores son en primer lugar eso: personas, que padecen y sienten igual que las de menor edad. Necesitan hablar, que les escuchen, salir a tomar algo... socializar, al fin y al cabo, como cualquier otra persona. Esta formación me ha quitado muchísimos de los prejuicios que tenía sobre los ancianos, como el de que siempre están de mal humor (la mujer a la que yo acompañaba era "la bomba")' (Participante 3).

APRENDIZAJE DE CONCEPTOS NUEVOS. Mencionaron el aprendizaje de conocimientos teóricos, relacionados con el tema de estudio (la soledad, las atribuciones causales, el acompañamiento, el voluntariado) y con los recursos y el voluntariado social de personas mayores, y, por otro lado, mencionaron el desarrollo de habilidades y competencias específicas que han tenido la oportunidad de practicar en el programa:

'Durante el curso hemos podido trabajar en equipo y aprender nuevas estrategias y recursos para tratar distintos tipos de situaciones. Hemos podido comprender aún más el mundo de la persona mayor, cómo puede sentirse y por qué a veces se comporta de una manera u otra. Me llevo un aprendizaje que seguro que pondré en práctica a lo largo de mi vida personal y profesional. Trabajando desde la empatía, la comprensión y llevando a cabo un estilo de comunicación asertiva, podremos alcanzar nuestros objetivos de manera más eficaz.' (Participante 12)

EXPERIENCIAS DIVERSAS DE APRENDIZAJE. En los discursos se mencionan los distintos escenarios y experiencias de aprendizaje que han podido experimentar a través del acompañamiento de la persona mayor, de las dinámicas grupales con las compañeras y de los ejercicios de reflexión entre sesiones. Asimismo, las participantes señalan haber aprendido de las personas mayores que han acompañado, produciéndose un aprendizaje vicario a partir de estos modelos de envejecimiento.

'He podido aprender cómo, aunque tiene sus limitaciones, sigue haciendo lo que le gusta y se mantiene activa ya que realiza multitud de actividades como viajar, está apuntada a cruz roja, está apuntada al colegio, ir al cine, etc.' (Participante 6)

CRECIMIENTO PERSONAL. Inicialmente siete personas mencionaron esta motivación y al finalizar el programa fueron 26 las participantes que reconocieron haber crecido personalmente. Por un lado, se hizo mención a un crecimiento personal vinculado a la mejora de aspectos personales (alcanzar mayor comprensión, madurez, empatía, autonomía, entre otros aspectos mencionados):

'Siento que he mejorado como persona después del acompañamiento' (Participante 1). 
'...me he hecho mucho más comprensiva y empática' (Participante 2).

Por otro lado, las participantes experimentaron estados afectivos positivos (experimentación de emociones secundarias como: contento, felicidad, bienestar y orgullo) relacionados con la experiencia de acompañamiento, sobre todo, manifestaron una gran satisfacción personal al haber participado y acompañado a la persona mayor, llegando a sentirse autorrealizadas tras el programa.

'Muy satisfecha porque no ha sido fácil. Ha sido un reto y creo que lo he llevado a cabo de la mejor manera que he sabido y estoy contenta con ello' (Participante 16).

'Muy realizada y contenta de alegrarle a la persona que acompañaba los días con mi presencia' (Participante 27).

MEJORA. Dos personas reconocieron entre sus motivaciones iniciales que acompañar podría servirles para sentirse mejor consigo mismas y ninguna de ellas buscaba conocer gente $\mathrm{O}$ establecer nuevas relaciones. Al finalizar el programa fueron 24 participantes las que satisficieron motivaciones de mejora, pero en estos casos las citas se centraron en destacar sentimientos positivos por haber conocido gente y haber podido establecer vínculos de amistad con la persona mayor y con las compañeras.
'Encantada, agradecida y afortunada, por poder haber conocido a A., además de sentir que no va a ser una despedida, sino que a $A$. y a mí nos queda una larga amistad y muchas actividades más por hacer' (Participante 4).

Tres personas se refirieron a una mejora autodirigida, es decir, afirmaban sentirse mejor consigo mismas o tener más autoestima tras participar en el programa.

'Me siento mejor conmigo misma y con ganas de hacer más actividades de este tipo' (Participante 19)

VALORES. Al inicio 11 participantes mencionaron que ayudar a otros, esto es, la solidaridad, era una de sus principales motivaciones para participar. Al finalizar el programa, ocho participantes (cinco de ellos diferentes a los anteriores) reconocieron que se había reforzado su valor de solidaridad y, además, mencionaron otros valores como el respeto y el amor.

'He aprendido que hay que ver las cosas positivas, la generosidad de M., la importancia de hacer el bien a los demás y ser buena persona, las experiencias que ha tenido y me contaba sobre lo importante que es también no querer quedar por encima de otro y dejarle mal' (Participante 21).

Tabla 4

Análisis temático de las motivaciones satisfechas en las participantes

\begin{tabular}{|c|c|c|c|c|c|}
\hline Tipo de motivación & $\begin{array}{l}\text { Motivación } \\
\text { (Temas) }\end{array}$ & $f$ (citas) & $f$ (muestra) & Subtemas & $\begin{array}{l}\text { Otros temas } \\
\text { destacados }\end{array}$ \\
\hline \multirow{4}{*}{$\begin{array}{l}\text { Heterocentrado } \\
\text { (23 citas) }\end{array}$} & Valores & 10 & 8 & $\begin{array}{l}\text { Solidaridad, amor y } \\
\text { respeto }\end{array}$ & \\
\hline & $\begin{array}{l}\text { Compromiso } \\
\text { social }\end{array}$ & 5 & 5 & $\begin{array}{l}\text { Pequeños gestos pueden } \\
\text { cambiar el mundo }\end{array}$ & \\
\hline & $\begin{array}{l}\text { Preocupación } \\
\text { comunitaria }\end{array}$ & 4 & 4 & $\begin{array}{l}\text { Valorar y atender mejor a } \\
\text { las personas mayores }\end{array}$ & \\
\hline & Reciprocidad & 4 & 4 & $\begin{array}{c}\text { La compañía ha sido } \\
\text { mutua }\end{array}$ & \\
\hline
\end{tabular}


Tabla 4 (Continuación)

Análisis temático de las motivaciones satisfechas en las participantes

\begin{tabular}{|c|c|c|c|c|c|}
\hline $\begin{array}{l}\text { Tipo de } \\
\text { motivación }\end{array}$ & $\begin{array}{l}\text { Motivación } \\
\text { (Temas) }\end{array}$ & $f$ (citas) & $\begin{array}{c}f \\
\text { (muestra) }\end{array}$ & Subtemas & Otros temas destacados \\
\hline \multirow{12}{*}{$\begin{array}{l}\text { Autocentrados } \\
\text { (170 citas) }\end{array}$} & \multirow{3}{*}{ Conocimiento } & \multirow[t]{3}{*}{102} & \multirow[t]{3}{*}{32} & $\begin{array}{l}\text { Sensibilización hacia las } \\
\text { personas mayores }\end{array}$ & Edadismo \\
\hline & & & & $\begin{array}{l}\text { Adquisición de } \\
\text { conceptos, habilidades y } \\
\text { competencias }\end{array}$ & $\begin{array}{l}\text { Soledad, atribuciones causales, } \\
\text { acompañamiento, voluntariado, } \\
\text { recursos para mayores, } \\
\text { habilidades sociales, flexibilidada, } \\
\text { resolución de problemas y otros. }\end{array}$ \\
\hline & & & & $\begin{array}{l}\text { Nuevas experiencias } \\
\text { con las personas } \\
\text { mayores, compañeras, } \\
\text { organizaciones }\end{array}$ & $\begin{array}{l}\text { Aprendizaje vicario, aprendizaje } \\
\text { reflexivo. }\end{array}$ \\
\hline & \multirow{4}{*}{$\begin{array}{l}\text { Crecimiento } \\
\text { personal }\end{array}$} & \multirow[t]{4}{*}{32} & \multirow[t]{4}{*}{26} & Satisfacción personal & \\
\hline & & & & $\begin{array}{l}\text { Desarrollo emociones } \\
\text { positivas }\end{array}$ & $\begin{array}{l}\text { Contento, felicidad, orgullo, } \\
\text { gratifificación, bienestar }\end{array}$ \\
\hline & & & & $\begin{array}{l}\text { Mejorar aspectos } \\
\text { personales }\end{array}$ & $\begin{array}{l}\text { Madurez, autonomía, empatía, } \\
\text { comprensión, intelecto, aprecio } \\
\text { por las pequeñas cosas }\end{array}$ \\
\hline & & & & Autorrealización & \\
\hline & \multirow[t]{2}{*}{ Mejora } & \multirow[t]{2}{*}{29} & \multirow[t]{2}{*}{24} & Conocer personas nuevas & $\begin{array}{c}\text { Amistad con la persona mayor y/o } \\
\text { compañeras; conocer a agentes } \\
\text { del voluntariado (coordinadora) }\end{array}$ \\
\hline & & & & Autoestima & \\
\hline & Disfrute & 3 & 3 & $\begin{array}{l}\text { Disfrutar con la persona } \\
\text { acompañada }\end{array}$ & \\
\hline & Carrera & 3 & 2 & $\begin{array}{l}\text { Interés por } \\
\text { especialización en } \\
\text { gerontología }\end{array}$ & \\
\hline & $\begin{array}{l}\text { Interés en la } \\
\text { tarea }\end{array}$ & 1 & 1 & $\begin{array}{l}\text { Interés por actividades } \\
\text { similares }\end{array}$ & \\
\hline
\end{tabular}

'A respetar la forma de pensar de la gente y que sigue habiendo gente buena en el mundo' (Participante 31).

\section{SATISFACCIÓN DE LAS MOTIVACIONES E INTERÉS HACIA EL VOLUNTARIADO}

El $84,8 \%$ de las participantes satisfizo más motivaciones de las que se plantearon al inscribirse al programa (Tabla 5), siendo un total de 23 las participantes que lograron satisfacer algunas de sus motivaciones iniciales con el curso (señaladas en negrita en la Tabla 5). En general se observaron niveles muy altos de satisfacción con el acompañamiento realizado y con la coordinación del programa, lográndose un nivel de satisfacción global con el programa excelente.

El $100 \%$ de las participantes reconocieron que sentían más interés en participar en algún tipo de voluntariado en el futuro. Al menos 14 participantes manifestaron que ya estaban mirando opciones para inscribirse en alguna acción de voluntariado y algunas de ellas 
Tabla 5

Motivaciones y satisfacción de las participantes

\begin{tabular}{|c|c|c|c|c|c|c|}
\hline $\begin{array}{l}\text { Partici- } \\
\text { pante }\end{array}$ & $\begin{array}{l}\text { Motivaciones } \\
\text { iniciales }\end{array}$ & $\begin{array}{l}\text { Motivaciones } \\
\text { satisfechas }\end{array}$ & $\begin{array}{l}\text { Satisfacción } \\
\text { motivaciones }\end{array}$ & $\begin{array}{l}\text { Satisfacción } \\
\text { tarea }\end{array}$ & $\begin{array}{l}\text { Satisfacción } \\
\text { coordinación }\end{array}$ & $\begin{array}{c}\text { Satisfacción } \\
\text { global }\end{array}$ \\
\hline $\mathrm{Pl}$ & 5 & 3,4 & No & 10 & 10 & 10 \\
\hline P2 & 4,12 & $3,4,7,12$ & Sí & 9 & 9 & 9 \\
\hline P3 & 3 & $3,4,7,12$ & Sí & 8 & 10 & 9 \\
\hline P4 & $3,4,12$ & $3,4,7$ & Sí & 10 & 10 & 9 \\
\hline P5 & 3,12 & 3,7 & Sí & 9 & 9 & 9 \\
\hline P6 & 5 & 3 & No & 10 & 10 & 10 \\
\hline P7 & 3 & $3,4,7,12$ & Sí & 7 & 9 & 10 \\
\hline P8 & $1,3,4$ & $1,3,7,12$ & Sí & 8 & 10 & 8 \\
\hline PQ & 1,3 & 3,8 & Sí & 8 & 10 & 9 \\
\hline P10 & 8 & $2,4,8,12$ & Sí & 9 & 9 & 8 \\
\hline P11 & 6,8 & $3,4,7$ & No & 10 & 9 & 9 \\
\hline $\mathrm{P} 12$ & 2,7 & $1,2,3,4$ & Sí & 8 & 10 & 9 \\
\hline $\mathrm{P} 13$ & 3,12 & $3,4,7$ & Sí & 10 & 9 & 10 \\
\hline P14 & 8 & 3,7 & No & 9 & 8 & 9 \\
\hline P15 & 8,12 & $2,3,4,7,12$ & Sí & 10 & 9 & 9 \\
\hline P16 & 3,4 & $3,4,5,7$ & Sí & 8 & 9 & 8 \\
\hline $\mathrm{P} 17$ & 12 & $3,5,7,10$ & No & 10 & 10 & 10 \\
\hline P18 & 3 & $3,4,7$ & Sí & 10 & 10 & 10 \\
\hline P19 & 7,8 & $3,4,6,7,10$ & Sí & 10 & 9 & 10 \\
\hline P20 & 4 & $3,4,7$ & Sí & 10 & 10 & 10 \\
\hline P21 & 3,12 & $3,4,12$ & Sí & 10 & 10 & 10 \\
\hline P22 & 3 & $3,4,7,10$ & Sí & 9 & 9 & 8 \\
\hline P23 & $3,4,12$ & $2,3,4,7$ & Sí & 10 & 10 & 9 \\
\hline P24 & 1 & 3,5 & No & 10 & 10 & 9 \\
\hline P25 & $3,4,6$ & 3,4 & Sí & 10 & 10 & 9 \\
\hline P26 & 12 & $3,4,7$ & No & 10 & 10 & 8 \\
\hline P27 & 1,12 & $3,4,7$ & No & 10 & 10 & 10 \\
\hline P28 & 3 & $3,4,7,10$ & Sí & 10 & 10 & 10 \\
\hline P29 & 2 & $3,4,7$ & No & 10 & 10 & 7 \\
\hline P30 & 1,8 & $3,4,7,8$ & Sí & 9 & 10 & 9 \\
\hline P31 & 1 & $3,4,7,12$ & No & 10 & 10 & 10 \\
\hline P32 & $3,5,8$ & $2,3,4,8$ & Sí & 10 & 10 & 10 \\
\hline P33 & 3,12 & $3,4,7$ & Sí & 10 & 10 & 9 \\
\hline Total & $N=58$ & $N=193$ & $\begin{array}{c}\mathrm{Si}=69,7 \% \\
\mathrm{No}=30,3 \%\end{array}$ & $\begin{array}{l}M=9,42 \\
D T=0,86\end{array}$ & $\begin{array}{l}M=9,63 \\
D T=0,54\end{array}$ & $\begin{array}{l}M=9,15 \\
D T=0,79\end{array}$ \\
\hline
\end{tabular}

Nota: los códigos de las motivaciones son $1=$ Carrera, $2=$ Compromiso social, $3=$ Conocimiento, $4=$ Crecimiento personal, $5=$ Disfrute, $6=$ Interés en la tarea, $7=$ Mejora, 8=Preocupación comunitaria, 9=Protección, 10=Reciprocidad, $11=$ Social y $12=$ Valores. 
informaron sobre el tipo de voluntariado por el que sentían interés: voluntariado social $(n=3)$, seguido de voluntariado medioambiental $(n=2)$, voluntariado educativo con niños $(n=1)$ y voluntariado con animales $(n=1)$.

En cuanto a la intención de realizar algún tipo de voluntariado de acompañamiento de personas mayores, el $97 \%$ confirmó que volvería a participar en alguna iniciativa similar. No obstante, algunas participantes $(n=7)$ matizaron qué condiciones se tendrían que dar para poder implicarse en este tipo de acción en el futuro: tener tiempo, ajustar el acompañamiento a un periodo del año que les venga bien para compatibilizarlo con el curso académico y procurar que el acompañamiento fuese próximo a su lugar de residencia.

\section{DISCUSIÓN}

Este estudio ha permitido conocer las motivaciones que impulsan al estudiantado universitario de la Generación Z a participar en acciones de formación en voluntariado con personas mayores, así como conocer en profundidad los principales beneficios que obtienen al participar en una experiencia de aprendizaje y servicio de acompañamiento a personas mayores que viven solas.

\section{MOTIVACIONES INICIALES}

En cuanto a las motivaciones que han impulsado a las participantes a realizar el Programa Acompaña-Té, se esperaba observar con mayor frecuencia motivaciones autodirigidas que heterodirigidas (hipótesis 1), tendencia que se ha confirmado en el estudio, donde las motivaciones autodirigidas orientadas a adquirir conocimientos, crecer personalmente, desarrollar competencias profesionales, disfrutar, mejorar y el interés en la tarea de acompañamiento han representado casi dos tercios de las citas analizadas. No obstante, si analizamos las motivaciones más frecuentes, se aprecia cierto balance entre los motivos auto y heterodirigidos, ya que la adquisición de conocimientos y los valores han sido, por ese orden, los motivos más citados. Las mismas tendencias se han observado en estudios anteriores con población universitaria (Chapman y Morley, 1999; Cho et al., 2018; Francis, 2011 ; Gillespie y King, 1985), donde los conocimientos, la carrera y los valores son las motivaciones fundamentales hacia el voluntariado. Lo cual es coherente con el contexto en el que se realiza la evaluación, con estudiantes en pleno desarrollo formativo y profesional.

Por otro lado, se ha observado que la opinión o influencia social de las personas importantes, la protección ante sentimientos negativos y el sentido de reciprocidad por dar lo que se ha recibido, no son motivaciones importantes para las participantes. Normalmente, la motivación social y de protección han sido las funciones menos importantes del modelo funcional en la literatura (Chapman y Morley, 1999; Francis, 2011), incluso se ha llegado a eliminar esta última en la evaluación de las motivaciones hacia el voluntariado en población universitaria (Cho et al., 2018). Sin embargo, con la motivación social ha habido más controversia. Por un lado, se ha afirmado que esta función tiene más peso entre los jóvenes (Kehl, Kietzmann y Schmidt, 2017), llegando a considerarla una motivación fundamental en la generación de millennials (Francis, 2011 1). En otra dirección, los estudios de Clary y Snyder (1999), con la generación $X$, muestran que es una función poco relevante $y$, recientemente, Cho et al. (2018), con la generación Z, han demostrado que la motivación social no aporta nada en la explicación de las actitudes hacia el voluntariado. Las tendencias observadas en el presente estudio, realizado también con estudiantes de la generación $Z$, van en la misma dirección, pues las participantes no han mencionado la motivación social ni al inicio ni al finalizar el programa. Estos resultados no implican que el estudiantado no esté motivado por las relaciones sociales, de hecho, en este estudio se ha observado que es uno de los principales beneficios que encuentran al participar en el programa, sino que la motivación inicial para participar en acciones de voluntariado con personas mayores no está influida por las personas significativas de su entorno. 
Sobre el número de motivaciones y la variedad de motivos iniciales referidos por las participantes, en la línea propuesta por Vecina y Marzana (2019) se esperaba observar pocos motivos y más variados de lo que se propone en la aproximación funcional de Clary y Snyder (1999), lo que se ha corroborado con un rango de motivos iniciales que oscilan entre uno y tres y un total de nueve motivaciones referidas, de las 12 motivaciones de Vecina y Marzana (2019). Asimismo, es relevante señalar que el número de motivaciones satisfechas fue mayor (de uno a cinco motivos mencionados) y en total se mencionaron 10 tipos de motivos distintos. El hecho de que no se hayan mencionado algunas de las motivaciones propuestas en la clasificación de Vecina y Marzana (2019), podría deberse al contexto de la evaluación, que se da en una población pequeña con estudiantado universitario. Según los datos analizados, los motivos de protección y social no son relevantes para esta población, lo que podría cuestionar la adecuación del modelo funcional del voluntariado en la evaluación de las motivaciones hacia el voluntariado en población universitaria.

\section{MOTIVACIONES SATISFECHAS}

Por otro lado, el Programa AcompañaTé ha sido diseñado con el objetivo de: 1) sensibilizar al estudiantado universitario sobre las personas mayores y la soledad que puede afectar a aquellas que viven solas (conocimiento); 2) crear experiencias de aprendizaje que favorezcan el vínculo entre personas de distintas generaciones (mejora: área social); y 3) promover la solidaridad intergeneracional (valores). Los resultados del análisis de contenido y el análisis temático sugieren que se han conseguido los beneficios esperados con el programa confirmándose así la segunda hipótesis del estudio. Además, se ha podido cuantificar en qué medida esto ha sido así y entender qué ha significado para ellas la consecución de estas motivaciones. No obstante a lo anterior, las participantes también han referido otros beneficios, entre los que ha destacado especialmente el crecimiento personal. Todos los beneficios positivos encontrados van en la línea de los referidos en otras experiencias de aprendizaje y servicio (Caire, 2019; Gershfeld-litvin, 2020; Martínez et al., 2020; Primavera, 1999; Saz y Ramo, 2015) y experiencias de voluntariado (Johnson et al., 1998; Popova y Popova, 2018) con estudiantes universitarios.

El análisis temático de las motivaciones satisfechas no ha estado exento de dificultades. Los conflictos han surgido, sobre todo, a la hora de clasificar algunas citas en los códigos de crecimiento personal y de mejora propuestos en la clasificación de Vecina y Marzana (2019). Estas autoras ampliaron el número de funciones hacia el voluntariado de la aproximación funcional (de seis a 12 motivaciones). Entre otras, crearon la categoría de crecimiento personal para diferenciarla de la mejora, que incluye narraciones sobre la mejora de la autoestima y la mejora de las relaciones sociales al hacer amigos (dos temas que posiblemente tampoco deberían considerarse parte de la misma función). Sin embargo, no aclararon conceptualmente las diferencias entre estos términos estrechamente relacionados.

El crecimiento personal, siguiendo el modelo de Ryff, sería una meta-dimensión referida al proceso de continuo desarrollo, fundamental para lograr la integración de diferentes aspectos del yo (Goñi, 2009), que implica aspectos recogidos en las dimensiones de mejora y crecimiento personal propuestas por Vecina y Marzana (2019). Por ejemplo, en el estudio de Cortés et al. (2017) se propone una escala para medir el crecimiento personal de estudiantes de medicina que está compuesta por 11 subescalas entre las que se encuentra, entre otras, la autoestima y el autoconocimiento y expresión emocional. En la misma línea, Aciego, Domínguez y Hernández (2005) observaron que el factor de autoconcepto saturaba fundamentalmente con factores de valoración personal, por lo que en el análisis factorial de segundo orden denominaron este factor como Autoconcepto y Autoestima, que recoge todos los aspectos de la valoración de uno mismo y la autoestima.

En este estudio se han identificado tres 
niveles de crecimiento personal en las participantes: 1) individual (yo y mi relación conmigo), que recoge aspectos sobre la imagen personal y el sentimiento de valía personal en relación con la imagen previa que se tenía de una misma; 2) relacional (yo y mi relación con los demás), que recoge narrativas sobre la oportunidad para conocer gente y establecer nuevos vínculos que devuelven una imagen nueva de sí mismas; y 3) funcional o de actuación (yo y mi relación con la tarea o acción desarrollada), que se refiere la imagen de una misma en relación a una acción o meta propuesta, esto incluiría aspectos como sentirse realizada o satisfecha por haber completado la acción de voluntariado de manera adecuada.

Las dificultades encontradas en la codificación, así como la literatura revisada sobre estos conceptos, podrían indicar que el modo de evaluar las motivaciones de mejora y crecimiento personal propuesto desde el enfoque funcional y las revisiones que se han hecho de este (Chacón et al., 2010; Clary et al., 1998; Clary y Snyder, 1999; Vecina y Marzana, 2019) no serían adecuadas para analizar específicamente los efectos del voluntariado en el crecimiento personal del voluntariado joven. Sería muy interesante que la investigación futura sobre el tema logre delimitar y aclarar mejor la evaluación de estas funciones del voluntariado.

\section{SATISFACCIÓN E INTERÉS EN EL VOLUNTARIADO}

En la tercera hipótesis se planteaba que las participantes que hubiesen obtenido beneficios funcionalmente relevantes, es decir, aquellas que satisficieron sus motivaciones iniciales, tendrían mayor nivel de satisfacción. Sin embargo, la puntuación de la satisfacción global media de las participantes que no satisficieron sus motivaciones iniciales fue mayor que aquellas que sí consiguieron satisfacer alguna de sus motivaciones iniciales. El enfoque funcional del voluntariado defiende la idea de que los voluntarios que alcancen sus motivaciones iniciales se sentirán más satisfechos y eso influirá en su permanencia en las organizaciones de voluntariado (Clary et al., 1998; Clary y Snyder, 1999; Jiménez et al., 2009; Vecina y Marzana, 2019). La tendencia observada aquí podría cuestionar esta idea, puesto que se ha observado que existen voluntarias que no han satisfecho sus motivaciones iniciales, pero tienen una alta satisfacción global y, además, tanto la intención de participar en acciones similares como el interés por realizar acciones de voluntariado en el futuro es casi unánime en todas las participantes. Esto podría deberse a que estas participantes, aunque no satisficieron su motivación inicial, sí obtuvieron otros beneficios significativos para ellas 0 , también, podría deberse a que son otros aspectos, como la tarea de voluntariado y la coordinación y gestión del programa, los que tienen más peso en su nivel de satisfacción global percibida con el programa, así como en su intención e interés en realizar acciones similares de voluntariado.

El tipo de estudio desarrollado aquí no permite examinar estas hipótesis, pero los análisis descriptivos realizados y la integración de datos cualitativos y cuantitativos sí ayudan a observar las principales tendencias en este sentido. En este estudio se ha creado una medida de correspondencias entre motivos iniciales y satisfechos para realizar la integración de la información. Cabe destacar que esta información ha sido extraída explícita y objetivamente de las narrativas de las participantes y, además, se ha realizado de manera indirecta, esto es, sin preguntar abiertamente a las participantes. De este modo, se puede considerar un dato más objetivo y válido, pues se evita la confirmación de las expectativas y la deseabilidad social de las participantes.

A la hora de evaluar la satisfacción de este tipo de programas preguntar directamente a las participantes tiene sus limitaciones. En este caso, aunque los cuadernillos de evaluación llevaban un código de identificación que ocultaba la identidad de la persona voluntaria, la información contenida sí podría vincularse con la persona acompañada y, por tanto, identificarle, pudiendo influir esto en sus valoraciones. Una solución para controlar 
este tipo de efectos en futuras ediciones sería hacer estas encuestas totalmente anónimas y separar la evaluación de estos aspectos (satisfacción, interés e intención de participar en acciones de voluntariado) del resto de variables.

El diseño mixto anidado secuencial empleado ha ayudado a comprender mejor los resultados de una experiencia de aprendizaje y servicio con personas mayores en una muestra de estudiantes jóvenes de la Generación Z. Los hallazgos y conclusiones derivados del mismo no se pueden generalizar, pero sí permiten comprender y conocer en profundidad cómo se nutren los estudiantes universitarios de este tipo de experiencias de aprendizaje y podrían servir para transferir y adaptar este tipo de experiencias a contextos similares (Mieles-Barrera, Toscano y Salgado, 2012).

Las implicaciones prácticas de este estudio son varias. Por un lado, los hallazgos descritos sugieren la viabilidad y los efectos positivos de desarrollar acciones formativas que promuevan el aprendizaje experiencial del alumnado en el contexto de la educación superior. Por otro lado, los resultados apuntan que para estimular la participación en este tipo de acciones educativas las universidades deben tener en cuenta las motivaciones que tienen los estudiantes para participar en estos programas, conectando la oferta formativa con los valores del estudiantado y con sus necesidades de formación y desarrollo profesional y personal. También, se ha observado que los beneficios que obtiene el estudiantado universitario al participar son múltiples: la adquisición de conocimientos, el crecimiento personal, la mejora de la red social y el reforzamiento de valores como la solidaridad han sido los efectos más relevantes de la experiencia con el Programa Acompaña-Té. Para que el alumnado universitario pueda obtener estos beneficios funcionales de la formación en voluntariado con personas mayores, es fundamental acompañar al alumnado durante su proceso de aprendizaje. Para ello sería recomendable ofrecer apoyo y guía desde la coordinación de estos programas con el objetivo de que el estudiantado culmine con éxito estas acciones y esté motivado por seguir aportando su granito de arena a la sociedad.

La experiencia de aprendizaje y servicio promovida con el Programa Acompaña-Té ha sido muy satisfactoria y enriquecedora para el estudiantado universitario que participó, dándoles la oportunidad de adquirir nuevos conocimientos y de transformarles personal y socialmente, haciéndoles más sensibles hacia las personas mayores, reconociendo no solo sus dificultades sino también sus potencialidades, generando, así, una visión más realista y menos estereotipada de este colectivo. Con acciones de este tipo se puede promover la formación integral del estudiantado universitario y desarrollar sociedades más inclusivas y solidarias.

\section{- Conflicto de intereses}

Los autores declaran no tener ningún conflicto de interés.

\section{REFERENCIAS}

Aciego, R., Domínguez, R., \& Hernández, P. (2005). Consistencia interna y estructura factorial de un cuestionario sobre autorrealización y crecimiento personal. Psicothema, 17(1), 134-142.

Beltrán-Llavador, J., Íñigo-Bajos, E., \& MataSegreda, A. (2014). La responsabilidad social universitaria, el reto de su construcción permanente. Revista iberoamericana de educación superior, 5(14), 3-18.

Blesio, M., \& Mendoza, M. (2016). La dimensión educativa y solidaria del voluntariado en la Universidad Nacional del Litoral. +E: Revista de Extensión Universitaria, 6, 164-171.

Braun, V., \& Clarke, V. (2006). Using thematic analysis in Psychology. Qualitative Research in Psychology, 3(2), 77-101. https://doi. org/10.1191/1478088706qp063oa

Brotons, P., Lorente, R., \& Sitges, E. (2020). Efectos del Programa ACOMPAÑA-TÉ sobre los estereotipos hacia la vejez en universitarios. Revista de Psicología de la Salud, 8(1), 5-43.

Caire, M. C. (2019). Contribuciones de las experiencias de aprendizaje-servicio desde la perspectiva de estudiantes de 
la Universidad Alberto Hurtado. RIDAS. Revista Iberoamericana de AprendizajeServicio, 7, 89-111. https://doi.org/ DOI10.1344/RIDAS2019.7.6

Chacón, F., Gutiérrez, G., Sauto, V., Vecina, M. L., \& Pérez, A. (2017). Volunteer functions inventory: A systematic review. Psicothema, 29(3), 306-316.

Chacón, F., Pérez, T., Flores, J., \& Vecina, M. L. (2010). Motivos del Voluntariado: Categorización de las Motivaciones de los Voluntarios Mediante Pregunta Abierta. Psychosocial Intervention, 19(3), 213-222.

Chapman, J. G., \& Morley, R. (1999). Collegiate service-learning: Motives underlying volunteerism and satisfaction with volunteer service. Journal of Prevention \& Intervention in the Community, 18(1-2), 19-33. https://doi. org/10.1300/J005v18n01 03

Cho, M., Bonn, M. A., \& Han, S. J. (2018). Generation Z's sustainable volunteering: Motivations, attitudes and job performance. Sustainability, 10(5), 1400. https://doi.org/10.3390/su10051400

Clary, E. G., \& Snyder, M. (1999). The Motivations to Volunteer: Theoretical and Practical Considerations. Current Directions in Psychological Science, 8(5), 156-159. https://doi. org/10.1111/1467-8721.00037

Clary, E. G., Snyder, M., Ridge, R. D., Copeland, J., Stukas, A. A., Haugen, J., \& Miene, P. (1998). Understanding and assessing the motivations of volunteers: $A$ functional approach. Journal of Personality and Social Psychology, 74(6), 1516 1530. https://doi.org/10.1037/0022. 3514.74 .6 .1516

Cortés, M. T., Petra, I., Acosta, E., Reynaga, J., Fouilloux, M., García, R., Piedra, E., Cortés, M. T., Petra, I., Acosta, E., Reynaga, J., Fouilloux, M., García, R., \& Piedra, E. (2017). Desarrollo y crecimiento personal. Construcción y validación de un instrumento para evaluar esta competencia en alumnos de medicina. FEM: Revista de la Fundación Educación Médica, 20(2), 65-73.

Escalante-Barrios, E. L., Herrón, M., Aguirre, C.E., \& Ferrer, M. A. (2020).
Métodos Mixtos en la Investigación Socioeducativa. En F. J. del Pozo (Comp.), Intervención educativa en contextos sociales: Fundamentos e investigación, estrategias y educación social en el ciclo vital (pp. 133-149). Colombia: Universidad del Norte.

Francis, J. E. (2011). The functions and norms that drive university student volunteering. International Journal of Nonprofit and Voluntary Sector Marketing, 16(1), 1-12. https://doi.org/10.1002/nvsm.390

Fundación Mutua Madrileña. (2020). VIII Estudio sobre Voluntariado Universitario (N.o 8). Fundación Mutua Madrileña. https://www.fundacionmutua.es/ actualidad/estudios/

Fundación Telefónica. (2019). Retrato del Voluntariado en España: Tendencias, experiencias innovadoras y cifras de un fenómeno creciente. Fundación Telefónica. https://www.sigmados.com/ wp-content/uploads/2019/11/Retratodel-Voluntariado-en-Espan\%cc\%83a.pdf Gershfeld-Litvin, A. (2020). Multi-learning experience course for psychology undergraduates: Combining experiential learning and theory-based research learning about disabilities. Journal of Disability Studies, 6(1), 36-39.

Gezuraga, M., \& García, Á. (2020). Recepciones de la pedagogía experiencial de Dewey en diversos enfoques metodológicos: El valor añadido del aprendizaje-servicio. Educatio Siglo XXI, 38, 295-316. https://doi.org/10.6018/ educatio.452921

Gillespie, D. F., \& King, A. E. O. (1985). Demographic Understanding of Volunteerism. Journal of Sociology and Social Welfare, 12, 798.

Goñi, E. (2009). El autoconcepto personal: Estructura interna, medida y variabilidad (Tesis Doctoral). Universidad del País Vasco/Euskal Herriko Unibertsitatearen, Victoria-Gasteiz. https://doi. org/10/12241

Jiménez, M. L. V., Fuertes, F. C., \& Abad, M. J. S. (2009). Satisfacción en el voluntariado: Estructura interna y relación con la permanencia en las organizaciones. Psicothema, 21 (1), 112 - 


\section{7.}

Johnson, M. K., Beebe, T., Mortimer, J.

T., \& Snyder, M. (1998). Volunteerism in adolescence: A process perspective. Journal of Research on Adolescence, 8(3), 309-332. https://doi.org/10.1207/ s15327795ira0803 2

Kehl, D., Kietzmann, D., \& Schmidt, S. (2017). Reasons for volunteering in the field of civil protection in Germany. Journal of Homeland Security and Emergency Management, 14(1). https:// doi.org/10.1515/ihsem-2016-0042

Landis, J. R., \& Koch, G. G. (1977). An Application of Hierarchical Kappa-type Statistics in the Assessment of Majority Agreement among Multiple Observers. Biometrics, 33(2), 363-374. https://doi. org/10.2307/2529786

Lorente, R., Brotons, P., \& Sitges, E. (2020). Estrategias para combatir el edadismo: ¿̇formación específica sobre envejecimiento $\bigcirc$ contacto intergeneracional? Cuaderno de Pedagogía Universitaria, 17(33), 6-16.

Martínez, V., Rodríguez, R. M., Marco, M., \& Macías, B. (2020). Percepciones del alumnado universitario sobre el aprendizaje-servicio como herramienta para su desarrollo personal y profesional. RIDAS. Revista Iberoamericana de Aprendizaje-Servicio, 9, 81-101. https:// doi.org/10.1344/RIDAS2020.9.5

Mieles-Barrera, M. D., Toscano, G. T. de, \& Salgado, S. V. A. (2012). Investigación cualitativa: El análisis temático para el tratamiento de la información desde el enfoque de la fenomenología social. Universitas Humanística, 74, 195-226.

Organización de las Naciones Unidas. (2015). Transformando nuestro mundo: La Agenda 2030 para el Desarrollo Sostenible (Resolución aprobada por la Asamblea General el 25 de septiembre de 2015 A/RES/70/1; p. 40). United Nations. http://undocs.org/A/RES/70/1

Organización Mundial de la Salud. (2016). Estrategia y plan de acción mundiales sobre el envejecimiento y la salud 20162020: Hacia un mundo en el que todas las personas puedan vivir una vida prolongada y sana (Resolución de la 69a
Asamblea Mundial de la Salud A69/A/ CONF./8; p. 5). Organización Mundial de la Salud. https://apps.who.int/gb/ ebwha/pdf files/wha69/a69 aconf8-sp. $\mathrm{pdf} ? \mathrm{va}=1$

Pérez-Díaz, J., Abellán-García, A., Aceituno-Nieto, P., \& Ramiro-Fariñas, D. (2020). Un perfil de las personas mayores en España 2020. Indicadores estadísticos básicos (N.o 25; Informes Envejecimiento en Red, p. 39). Consejo Superior de Investigaciones Científicas (CSIC). Centro de Ciencias Humanas y Sociales (CCHS). Envejecimiento en red. http://envejecimiento.csic. es/documentos/documentos/enredindicadoresbasicos2020.pdf

Popova, N. V., \& Popova, E. V. (2018). Volunteering as a factor in the formation of moral standards among young people. Obrazovanie i Nauka, 20(10), 139-155. https://doi.org/10.17853/1994-56392018-10-139-155

Primavera, J. (1999). The unintended consequences of volunteerism: Positive outcomes for those who serve. Journal of Prevention \& Intervention in the Community, 18(1-2), 125-140. https:// doi.org/10.1300/J005v18n01 10

Ruiz-Corbella, M., \& Bautista-Cerro, M. J. (2016). La responsabilidad social en la universidad española. Teoría de la Educación. Revista Interuniversitaria, 28(1), 159-188. https://doi. org/10.14201/teoredu2016281159188

Salazar, R. (2016). Los pilares para la educación superior del futuro: Responsabilidad social, calidad académica y servicio-aprendizaje (S-A). Fides et Ratio - Revista de Difusión cultural y científica de la Universidad La Salle en Bolivia, 17 (11), 155-179.

Saz, M. I., \& Ramo, R. M. (2015). Aproximación a los impactos y beneficios del aprendizaje servicio en la Universidad de Zaragoza. RIDAS. Revista Iberoamericana de Aprendizaje-Servicio, 1, 9-27.

Schroer, W. J. (2008). Defining, managing, and marketing to Generations $X, Y$ and $Z$. The Portal, XL(March/April), 9-10.

Sitges, E., Lorente, R., \& Saorín, M. J. 
(2017). Promoción y formación del Vecina, M. L., \& Marzana, D. (2019). voluntariado con personas mayores en Motivations for volunteering: Do la universidad española. International motivation questionnaires measure what Journal of Developmental and Educational actually drives volunteers? TPM - Testing, Psychology: INFAD. Revista de Psicología, 2(2), 99-116. https://doi.org/10.17060/ ijodaep.2017.n2.v2.1083

Stukas, A. A., Hoye, R., Nicholson, M., Brown, K. M., \& Aisbett, L. (2016). Motivations to Volunteer and Their Associations With Volunteers' WellBeing. Nonprofit and Voluntary Sector Quarterly, 45(1), 112-132. https://doi. org/10.1177/0899764014561122 